\title{
Intelligent Guidance Commands Technique Between two Autonomous Wheeled Mobile Robots
}

\author{
Mohammed Majid M. Al-Khalidy ${ }^{1}$, Ahmed H. Maki ${ }^{2}$, Ali A. Rasool ${ }^{3}$, Ahmed A. Rasool ${ }^{4}$ \\ Assistant Professor, Electrical and Electronics Department, College of Engineering, University of Bahrain, Bahrain ${ }^{1}$ \\ Electrical and Electronics Department, University of Bahrain, Bahrain ${ }^{2,3,4}$
}

\begin{abstract}
This paper proposes intelligent Guidance Commands technique between two autonomous Wheeled Mobile Robots (WMRs). The paper indicates the development of the principle of wireless communication commands and automatic control as a small prototype for the intelligent Electric Vehicle EVs (car that thinks). This achieved via designed of two autonomous WMRs, one of them equipped with robotic manipulator arm. The two robots communicate with each other's reciprocally through exchanging information and instructions. This leads to prevent vehicles from crashing and without the intervention of a human. The robots can work in the same place without any intervention of simultaneous tasks. This work aims to develop the idea of automatic car control and communication that allows the users to use these features safely and comfortably, also it can be used in Industrial automation or in a rescue cars for the areas that affected by natural disasters where it is impossible to reach. This technology enables these rescue robots that equipped with robotic manipulator arms working simultaneously together as a team to access the disasters areas and helping there. In the same time it can sends adequate vision information about the situation. The results preformed from this technique demonstrated the suitability of this methodology for safety in road and construction field.
\end{abstract}

Keywords: WMRs, Multi Sensors, Intelligent Communication Guidance technique, Fuzzy Controller.

\section{INTRODUCTION}

Recently, the universal competition in developing intelligent and effective robotics is high. A huge businesses have been started to manufacture smart interactive robots specially after Japan nuclear disaster in 2011 and they are still developing this technology.

The idea of making two WMRs interactive with each other without the intervention of a human can be done via precision sensors platform with active communication system. This will add an advantage of sending - receiving instructions in a way that making the robots can work in the same place without intervention of simultaneous tasks. Ögren et al., they have shown how to control a mobile sensor network to perform a gradient climbing task in an unknown, noisy, distributed environment. there result was the partial decoupling of the formation stabilization problem from the gradient climbing mission [1]. Zhang and Leonard, developed a cooperative Kalman filter that combines measurements from a small number ofmobile sensor platforms to cooperatively explore a static planar scalar field. They took a geometric approach information control where reduction was performed on the total configuration space of the formation using Jacobi vectors [2].

Guidance systems is one of the most important technologies that is used to improve the performance of the vehicle's mission regarding to its precision. Guidance systems are used with different fields like; military, industrial, agriculture, traffic control and robotics. All these fields shared in dividing the guidance systems in to two main types passive and active, but each one of these fields has its own way of implementing depending on the application that will be used. Diveev and Shmalko, formulated mathematically the problem of control synthesis by constructed a control function that autonomously provides control commands upon the received values of the object's state. The goal of synthesis is to find a multidimensional control function that depends on the current states of all objects [3].

Using two cooperatives' WMRs with high functionality gives more capability for these robots to finalize the task. E. A. Lee and S. A. Seshia, in their book they gave some imagination ideas through examples. One of their ideas was considering a city where traffic lights and cars cooperate to ensure efficient flow of traffic. In particular, imagine never having to stop at a red light unless there is actual cross traffic. Such a system could be realized with expensive infrastructure that detects cars on the road. But a better approach might be to have the cars themselves cooperate. They track their position and communicate to cooperatively use shared resources such as intersections [12]. For this the intersection was one of the problems that google car second realize faced. 


\section{International Journal of Innovative Research in Electrical, Electronics, Instrumentation and Control Engineering}

\section{ISO 3297:2007 Certified}

Vol. 5, Issue 5, May 2017

Serbencu, used two wheeled mobile robots (WMRs) equipped with robotic manipulators to assembly/disassembly Production Line. He proposed a model for a reversible assembly line useful in order to analyze its structural properties [4]. Lu et al., developed a constrained fast source seeking algorithm using two nonholonomic mobile robots. Slidingmode-like control algorithm is developed to generate the bounded linear velocities so that the moving directions of the two robots are aligned with the field gradient direction as quickly as possible. They desired a formation control module that represents their scenario in which the two robots should move in parallel and keep a constant distance between each other [5].

The performance of steering configuration play a main role in autonomous vehicle. There are three widely used types of steering configuration for four wheels vehicle which are; Ackerman, Differential and Skid. Ackerman steering configuration depends on the difference of angles between steering wheels during vehicle turning. Differential steering configuration use independent velocities in both rear wheels left and right. Skid steering configuration varying the velocities of the two wheels in the same line, which can vary the trajectory that the vehicle takes [6]. Some previous studies discussed these configurations. Wang et al., developed an analysis and experimental kinematics scheme of a skid-steering wheeled vehicle based-on a laser scanner sensor, their results show that there is a relationship between the instantaneous centers of rotation coefficient and a nondimensional path curvature variable parameter [7]. Wu et al., they showed the benefits of the independent motor driving for electric vehicle and studied the differential speed steering. They simulated and verified through results the rationality of control strategy for differential speed steering in four inwheel motors independent drive vehicle [8]. Al Khalidy, presented a novel kinematic model depending on a mutual steering configuration between differential steering wheels and Ackerman steering wheels[9]. SašoBlažič, presented a novel kinematic model where the transformation between the robot posture and the system state is bijective [13].

\section{STEERING CONFIGURATION}

This work depended on skid-steering configuration for both WMRs where, each pair of wheels are connected by the same line, which makes the robot turns at the same direction and speed as shown in Fig.1.

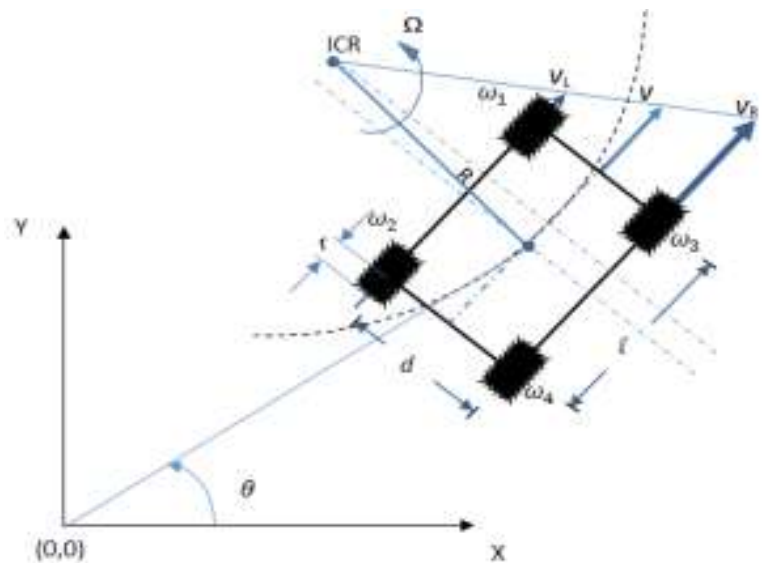

Fig. 1.Schematic Kinematics model for skid-steered vehicle

Now, if we consider $\mathrm{q}$ as a robot posture in the base frame then, $\dot{\mathrm{q}}$ denotes the vector of generalized velocities.

$$
\begin{gathered}
\mathrm{q}=\left[\begin{array}{c}
\mathrm{X} \\
\mathrm{Y} \\
\dot{\theta}
\end{array}\right]_{(1)} \\
\dot{\mathrm{q}}=(\dot{\mathrm{X}}, \dot{\mathrm{Y}}, \dot{\theta})^{\mathrm{T}}(2)
\end{gathered}
$$

The rotation matrix which express the orientation of the base frame with respect to the moving frame is:

$$
R_{\theta}=\left[\begin{array}{ccc}
\cos \theta & -\sin \theta & 0 \\
\sin \theta & \cos \theta & 0 \\
0 & 0 & 1
\end{array}\right] \text { (3) }
$$

Then, the robot velocities with the orientation $\theta$ in $\mathrm{X}, \mathrm{Y}$ frame will be:

$$
\left[\begin{array}{c}
\dot{X} \\
\dot{Y} \\
\dot{\theta}
\end{array}\right]=\left[\begin{array}{ccc}
\cos \theta & -\sin \theta & 0 \\
\sin \theta & \cos \theta & 0 \\
0 & 0 & 1
\end{array}\right]\left[\begin{array}{l}
\mathrm{v}_{\mathrm{x}} \\
\mathrm{v}_{\mathrm{y}} \\
\mathrm{w}_{\mathrm{z}}
\end{array}\right] \text { (4) }
$$


Vol. 5, Issue 5, May 2017

Now, for the angular velocities $\left(\varpi_{L}, \varpi_{R}\right)$, taking the assumption of:

$$
\begin{aligned}
& \varpi_{\mathrm{L}}=\omega_{1}=\omega_{2}(5) \\
& \varpi_{\mathrm{R}}=\omega_{3}=\omega_{4}(6)
\end{aligned}
$$

Then, the corresponding linear and angular velocities of the vehicle can be given by:

$$
\begin{gathered}
v=\frac{v_{R}+v_{L}}{2}=\frac{\sigma_{R}+\varpi_{L}}{2} r(7) \\
\varpi=\frac{v_{R}-v_{L}}{d}=\frac{\sigma_{R}-\sigma_{L}}{d} r(8)
\end{gathered}
$$

where, dis the separation distance of the two central wheels. The angular velocities of the wheels can be obtained by:

$$
\begin{gathered}
\varpi_{L}=\frac{v-(d / 2) \varpi}{r}(9) \\
\varpi_{R}=\frac{v+(d / 2) \varpi}{r}(10)
\end{gathered}
$$

The turning radius $\mathrm{R}$ with the assumption of no slippage, can be calculated as [10]:

$$
R=\frac{d}{2}\left(\frac{v_{L}+v_{R}}{v_{L}-v_{R}}\right)(11)
$$

Then, the turn angular velocity can be obtained from:

$$
\Omega=\frac{v_{L}+v_{R}}{2 R}(12)
$$

\section{III.CONTROL AND GUIDANCE METHODOLOGY}

Motion control might not be an easy task for autonomous robot, and it has to acquire knowledge about surrounding environment. This is done by taking measurements using various sensors and then extracting meaningful information from those measurements [11]. Visual servo control using machine vision that gathers information about the environment playing a major role in automation guidance system. Starting from fetching an image, image processing like locating object from the image and ending with sending the result to the automation guidance system to take an action. The Cyber-Physical Systems CPS mechanism of an autonomous WMR consists of three main blocks as shown in Fig. 2.

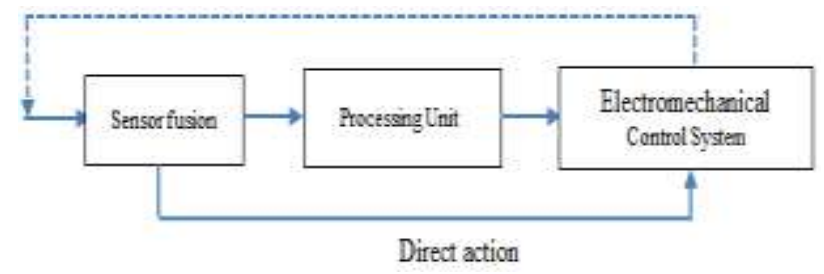

Fig. 1. Block diagram for autonomous WMR control mechanism

In this paper the desired control module depends on its design on a scenarios that the two robots will moves in different paths and different distance as shown in Fig. 3.

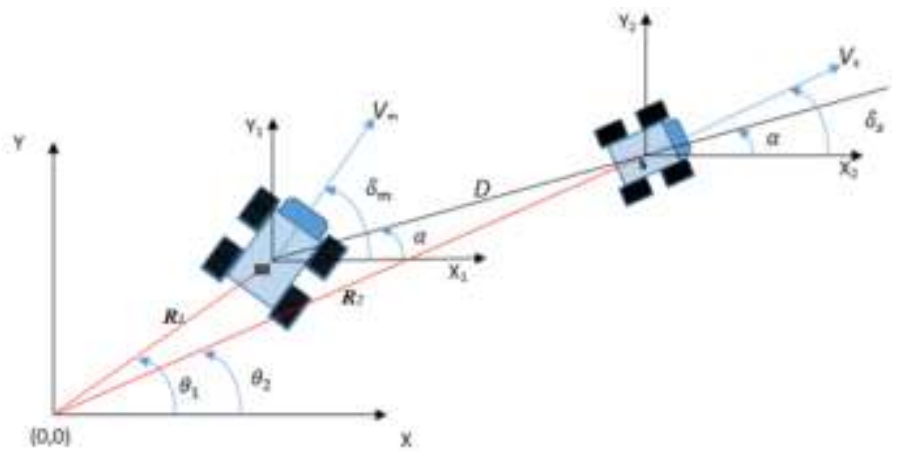

Fig. 2.Two Wheeled Mobile Robots in X,Y plane 


\section{IJIREEICE \\ International Journal of Innovative Research in Electrical, Electronics, Instrumentation and Control Engineering \\ ISO 3297:2007 Certified \\ Vol. 5, Issue 5, May 2017}

It is important to mention here that the information of the localization will be included but not the global position information. From Fig. 3, the actual distance D represent the distance between the two robots which can be derived as :

$$
\begin{gathered}
D=v_{s} \sin \emptyset_{1}-v_{m} \sin \emptyset_{2}(13) \\
\emptyset_{1}=\delta_{s}-\alpha(14) \\
\emptyset_{2}=\delta_{m}-\alpha(15)
\end{gathered}
$$

Where, $\emptyset_{1}$ is the angle between Vm and the line-of-sight LOS.And, $\emptyset_{2}$ is the angle between Vs and the line-of-sight LOS.

While the velocity component along the line of sight can be found from:

$$
\dot{D}=v_{s} \cos \emptyset_{1}-v_{m} \cos \emptyset_{2}(16)
$$

Where, $v_{m}$ and $v_{s}$ are master and slave robots velocities, $\delta_{s}$ and $\delta_{m}$ are the slave and master driving path angles and $\alpha$ is the line of sight angle. Practically, the distance D can be detected through the LiDar, Laser, IR or ultrasonic sensor. Now by considering the geometry that given in Fig. 3.; the position of the two robots regarding to the reference line component can be found as:

$$
\begin{gathered}
\dot{X}_{1}=v_{m} \cos \delta_{m}(17) \\
\dot{Y}_{1}=v_{m} \sin \delta_{m}(18) \\
\dot{X}_{2}=v_{s} \cos \delta_{s}(19) \\
\dot{Y_{2}}=v_{s} \sin \delta_{s}(20)
\end{gathered}
$$

By taking the integration for Eq. 17, 18, 19 and 20, then the $\mathrm{X}$ and $\mathrm{Y}$ distance components between the master robot and the slave robot can be found as:

$$
\begin{aligned}
& D_{s m_{x}}=X_{2}-X_{1}(21) \\
& D_{s m_{y}}=Y_{2}-Y_{1}(22)
\end{aligned}
$$

Then, the line of sight angle $\alpha$ is:

$$
\alpha=\arctan \frac{D_{s m_{y}}}{D_{s m_{x}}}(23)
$$

Considering that the demand is to make the two robots approaching toward each other and always the slave robot is the one who will follow the master robot, then the closing velocity Vc can be defined as :

$$
V_{c}=-\dot{D}(24)
$$

Now if the slave robot is approached toward the master robot then the slave robot acceleration guidance command is:

$$
\mathrm{A}_{\mathrm{cs}}=\mathrm{kV}_{\mathrm{c}} \dot{\alpha}(25)
$$

In the same way, if the master robot is approached toward the slave robot then the master robot acceleration guidance command is:

$$
\mathrm{A}_{\mathrm{cm}}=\mathrm{kV}_{\mathrm{c}} \dot{\alpha}(26)
$$

where, Acm and Acs is the master and slave robot acceleration guidance command, $\mathrm{k}$ represent the guidance constant gain, $\dot{\alpha}$ is the LOS angle rate.

Now, to be sure that there is a safety distance between the two robots a tracking closing distance $\mathrm{C}_{\mathrm{d}}$ is formed to be identical to the practical scenarios and its value is effective as one of the control signals:

$$
C_{d}=D-D_{0}(27)
$$

Where,

$$
\left\{\begin{array}{lll}
A_{\mathrm{cs}}=\mathrm{kV}_{\mathrm{c}} \dot{\alpha} & \text { when, } & \mathrm{C}_{\mathrm{d}}>\mathrm{D}_{\mathrm{o}} \\
0 & \text { when, } & \mathrm{C}_{\mathrm{d}} \leq \mathrm{D}_{\mathrm{o}}
\end{array}\right\}(28)
$$

If the master robot is the one who will approach to the slave robot then:

$$
\left\{\begin{array}{lll}
\mathrm{A}_{\mathrm{cm}}=\mathrm{kV}_{\mathrm{c}} \dot{\alpha} & \text { when, } & \mathrm{C}_{\mathrm{d}}>\mathrm{D}_{\mathrm{o}} \\
0 & \text { when, } & \mathrm{C}_{\mathrm{d}} \leq \mathrm{D}_{\mathrm{o}}
\end{array}\right\}(29)
$$

Where, $D_{o}$ is a desired safety distance between the two robots, The desired values of $D_{0}$ will set depending on a specific demand actions in the scenario. 


\section{IV.INTELLIGENT GUIDANCE COMMANDS TECHNIQUE}

The In the field of autonomous mobile robotics, more than one type of classification for communication system are used. Some of these classifications depend on a specific protocol, others depend on the way of communication.

Robot Guidance Commands (RGC) is the transfer of information and instructions between robots. The main idea of RGC is to make cooperativecommunication between robots to be possible and guide the robots towards the demand destination. In fact in some situations there is no need to transfer information between the robots since they are provided with a rigid sensors with an effective intelligent decision system. In this situation communicate for guidance commands instructions between robots is the task that each robot has to implement. This what was constructed in this paper with two different scenarios to show the effectiveness of intelligent RGC.

This paper proposed a solution for the road intersection problem that could be used for the intelligent EVs. The technique is done depending on a MIMO Global Fuzzy Controller GFC to produce Intelligent RGC for $\bar{\omega}_{\mathrm{L}}$ and $\bar{\omega}_{\mathrm{R}}$, in addition to communication commands and arms commands as illustrated in Fig. 3. The proposed technique has some constraints and assumptions as it mentioned in the next simulation part.

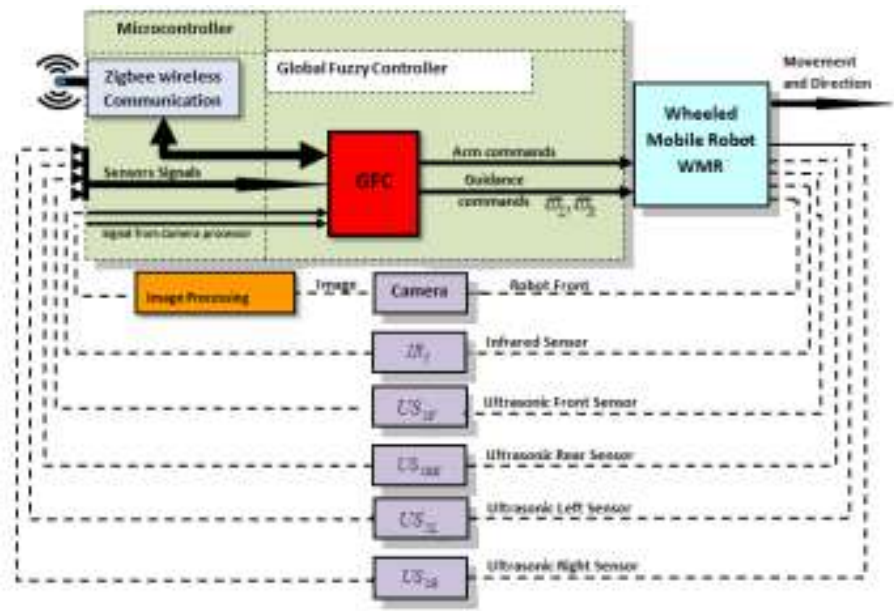

Fig. 4.Intelligent Control and Guidance Technique

\section{SIMULATION}

A realistic simulation to validate the effectiveness of the performance of the proposed technique has been verified. But before that a number of tests had been done as follow:

\section{A. Turning Test}

As the dimensions for each WMR were different, a turning test was done by using Matlab simulation. The simulation results obtained, showed a difference in turning radius $\mathrm{R}$ regarding to the difference in turning angle values between the two WMRs. as shown in Fig. 4 and 5. The dimensions for the master robot are: $r=0.05 \mathrm{~m}, \mathrm{~d}=0.30 \mathrm{~m}, \mathrm{l}=0.40 \mathrm{~m}$ and the dimensions for the slave robot are: $\mathrm{r}=0.03 \mathrm{~m}, \mathrm{~d}=0.12 \mathrm{~m}, \mathrm{l}=0.27 \mathrm{~m}$. And for simulation purpose we chose the angular velocity as: $\varpi_{\mathrm{L}}=\omega_{1}=\omega_{2}=0, \varpi_{\mathrm{R}}=\omega_{3}=\omega_{4}=3 \mathrm{rad} / \mathrm{s}$.

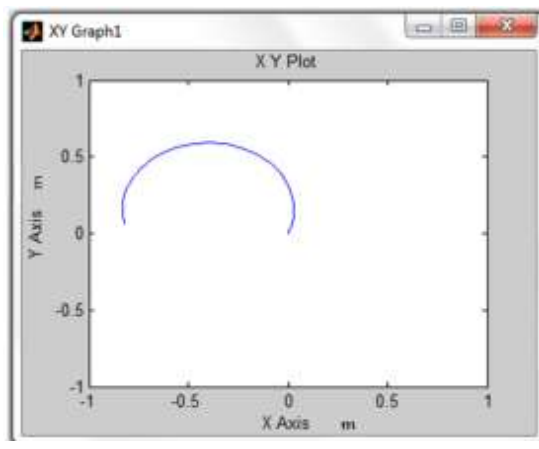

a

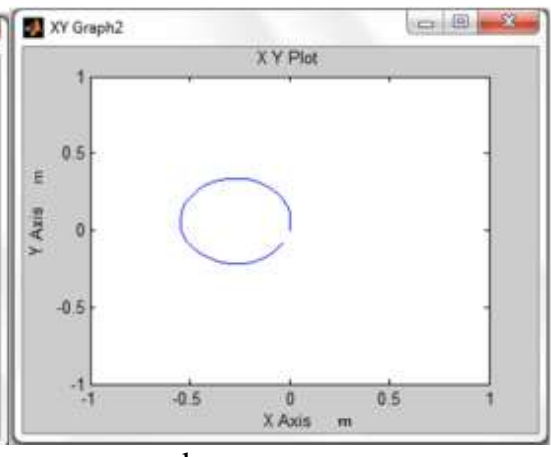

b

Fig. 5.a. Turning test for master WMR b. Turning test for slave WMR 


\section{International Journal of Innovative Research in Electrical, Electronics, Instrumentation and Control Engineering}

\section{ISO 3297:2007 Certified}

Vol. 5, Issue 5, May 2017

B. Manoeuvring test

By applying equal values for angular velocity to both WMRs, asecond test was done to check the capability of Manoeuvring for each WMR. The results showed a different in manoeuvring between the two robots. This difference came from the difference in dimensions between the two robots.
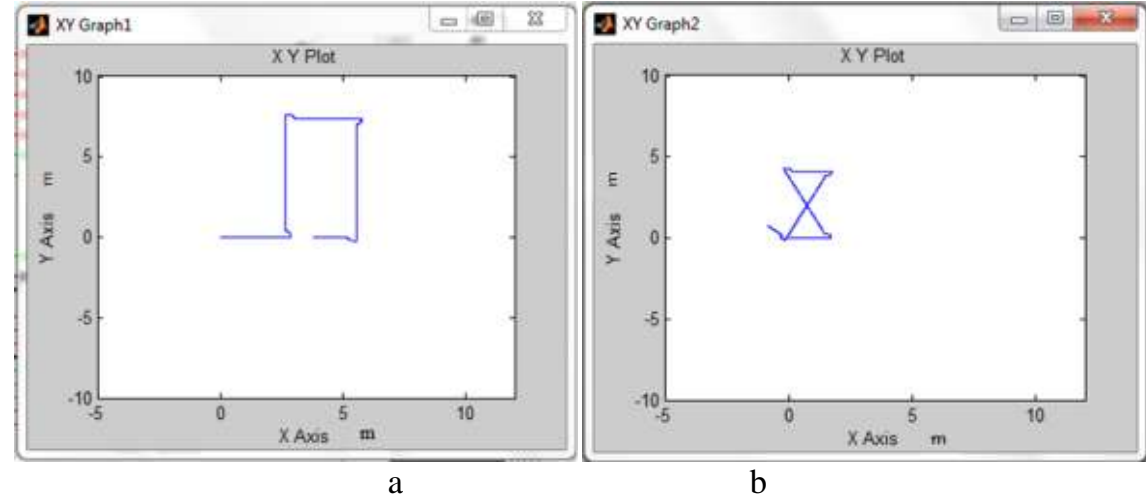

Fig. 6 a. Manoeuvring test for master WMR b. Manoeuvring test for slave WMR

C. Assumptions ofImplementation

A few assumptions considered to show the ability of WMRs to reach the required distention.

- Scenarios: Two desired scenarios were done, the first scenario represents road intersection. The second scenario composed of two mazes. The first maze is small to let only the small robot entering through it. The second maze is big to let the big robot entering through it.

- Robots: In both scenarios we used two WMRs. The big robot is the master robot which equipped with manipulator arm, sensors and camera. The small one is the slave robot that has a sensors platform only.

- Localizing: The path is a black line follower supported by a landmarks instead of using GPS within the real cars just to prove the powerful of RGC technique.

- Obstacles: In both scenarios the WMRs cared about avoid the obstacles and collision, and in some situations the master robot has to remove some obstacles by using the equipped manipulator arm.

- Communication: The greater communication concern was about the guidance commands through the wireless communication between the two robots, rather than the robots and the path.

- The intelligent wireless steering commands in this work was implemented by using XBee sensors for each robot which allowed them to uniquely identify each other and transfer intelligent RGC for navigation and to reach the event location. The Xbee is a wireless communication module based on Zigbee standard protocol. The Xbee allows communicating two or more intelligent devices with each other. The major considerations that made us use the Xbee are the Low cost, Flexibility, and Security. The Zigbee standard is a wireless communication based on IEEE 802.15.4- specification; the Zigbee has range longer than the Bluetooth range and power less than the Wi-Fi. Zigbee networks are secured by 128 bit symmetric encryption keys.

For the commands correspondence between the robots XBee series 2 wireless transceivers from Digiwere used. The maximum range of series 2 models is 40 meter indoor and 120 meter outdoor, at $2.4 \mathrm{GHz}$ frequency transmitting with $250 \mathrm{kbps}$ RF Data Rate. We chose this model to correspond the multi robots because of its specifications mainly; Pointto-point, Point-to-multipoint, Peer-to-peer and Mesh.

\section{The First Scenario}

The first scenario that implemented was the roads intersection as shown in Fig. 7, where the slave robot is located on the horizontal path, and the master robot is located on the vertical path. The autonomous WMRs must cross the intersection without collision. The slave robot in the horizontal path intersection has the highest priority to pass the intersection without unnecessary stopping. The master robot in the vertical path has to stop before passing the intersection regarding to the landmark (Give Way) that installed beside the intersection.

The technique of using landmark like "Give Way" is one of the scenario that works with traffic signs in the real world. Then the master robot will check if there is any robot in the horizontal path near the intersection. If there is a robot in the horizontal path, then the master robot will keep stopping and sending a pass confirmation to the slave robot to pass. Then the master robot will move as illustrated in Fig. 8. 
Vol. 5, Issue 5, May 2017

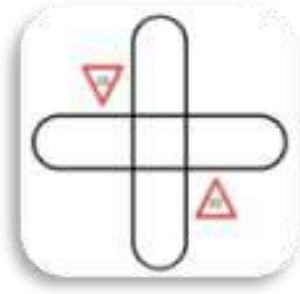

(a)

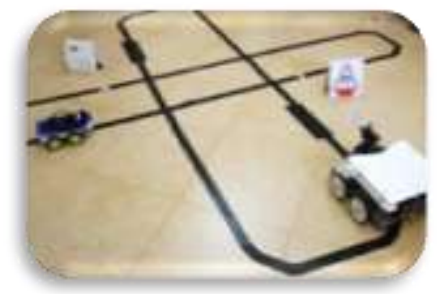

(b)

Fig. 7.a. The desired scenario for the intersection b. Both robots on the path before the intersection

Fig. 9. a. Shows the implemented of the first scenario. From the figure it can be recognized that WMRs are on the desired path. The master robot detected the "Give way" traffic sign through the camera sensor and stopped because the priority here is to the slave robot. The slave robot passed the intersection after detected that the path was clear, at the same time it received a conformation command from the master robot to move. Fig. 9. b. shows the master robot was movingthrough the intersection after the past of the slave robot.

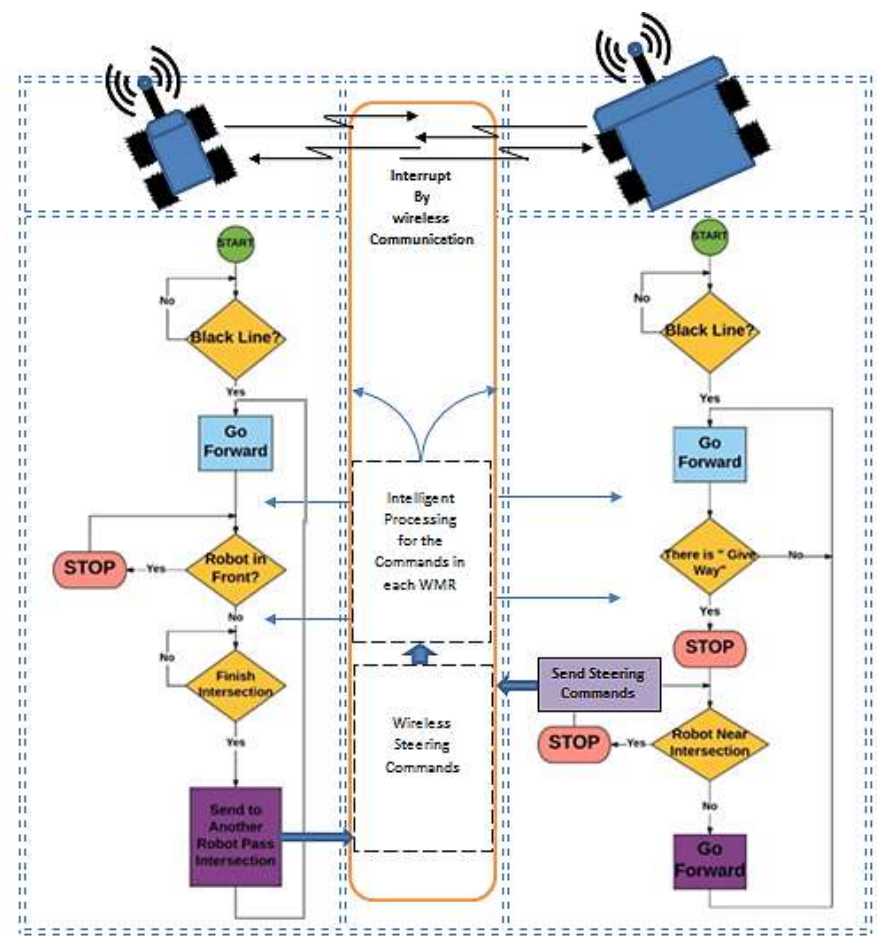

(a)

(b)

(c)

Fig. 8.Scenario (1), Command guidance flowchart a. Flow Chart for the Slave robot program b. Wireless Commands c. Flow Chart for the master robot program.
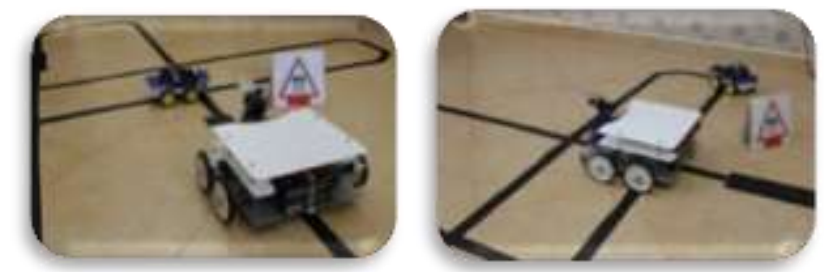

(a) (b)

Fig. 9.a. The slave robot passing the intersection and the master robot is waiting b. The master robot passing the intersection

The implementation of scenario 1. shows that the proposed intelligent RGC technique was effective and it can be applied in the real intersection roads specially for the driverless vehicles, and this technique will be faster and safer than the driver vehicles because there is no need for the intervention of human mind. 


\section{E. The Second Scenario}

The second scenario shows the capability of the proposed intelligent RGC technique to steer the WMRs through different surrounding situations. The second scenario composed of two mazes. The first maze is narrow $15 \mathrm{~cm}$ width to let only the slave robot to enter through it. The second maze is wide $50 \mathrm{~cm}$ width to let the master robot to enter through it. as shown in Fig. 10.

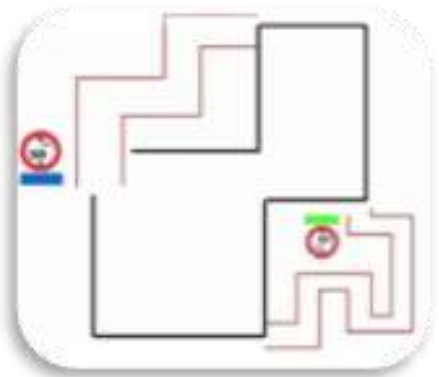

Fig. 10. a. Scenario (2) Desired scenario for the Synchronize autonomous robots

The requirements and constraints for the second scenario are:

- The scenario has to start by the movement of the master robot.

- The slave robot has to follow the master robot.

- If there is any obstacle in front of the master robot, then he has to avoid it.

- If there is an object then the master robot has to remove it or lade the object to a specific area by using the manipulator arm. In the same time sending a command to the slave robot to wait.

- After removing the object, the master robot has to continue following the path (black line).

- If the master robot detect the "15 cm " sign this means it is not allowed to the master robot to pass throw this zone, because it is a narrow area. So the master robot has to send a command to the slave robot to pass through this zone. Meanwhile the master robot has to continue tracking the path, until the slave robot passes the zone. The master robot will wait the slave robot at the end of the zone.

- If the master robot detect the "50 cm " sign, this means it is allowed for the master robot or both WMRs to pass throw this zone. The master robot has to pass through this zone and sends a command to the slave robot to follow him.

Fig. 11. Shows the guidance flowcharts for the second multifunction scenario for both WMRs regarding to the specific requirements and constrains.

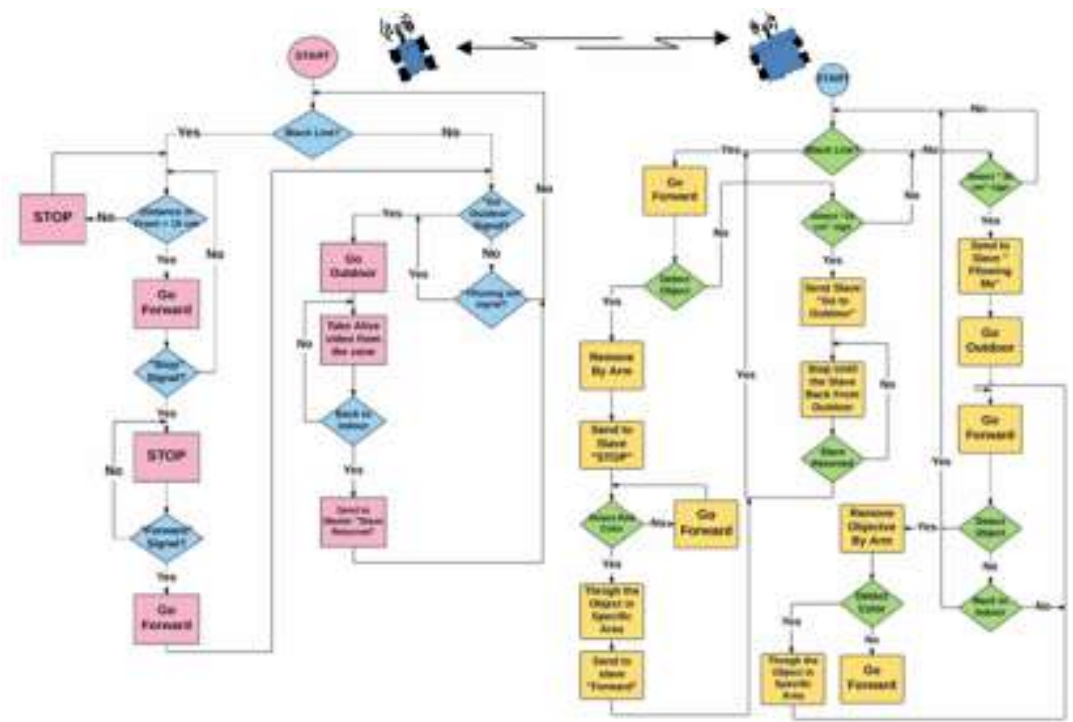

(a)

(b)

Fig. 11. Guidance flowchart- scenario (2) a. Flowchart for the slave robot program b. Flowchart for the master robot program. 
Fig. 12. Shows the implementation of the second scenario. It can be recognized that the WMRs are on the desired path.

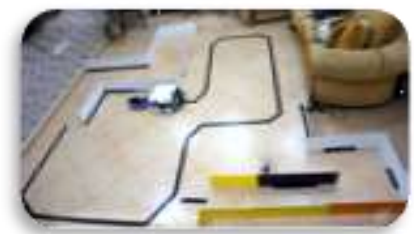

(a)

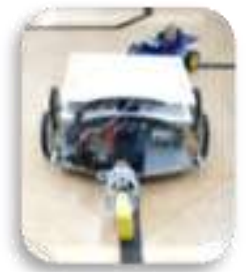

(c)

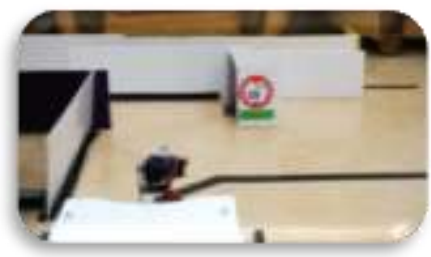

(f)

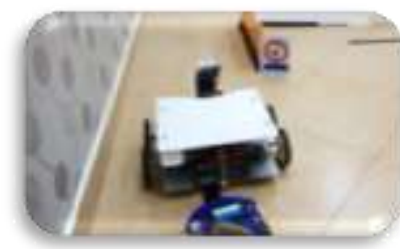

(h)

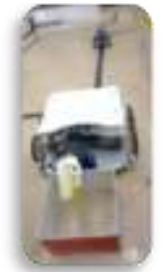

(d)

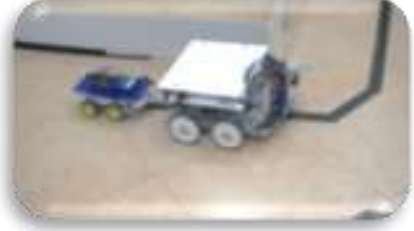

(b)

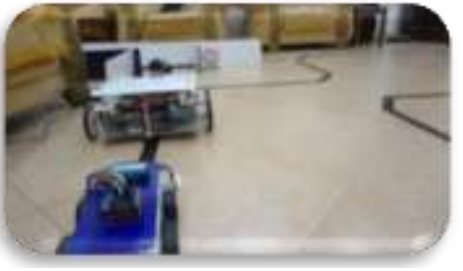

(e)

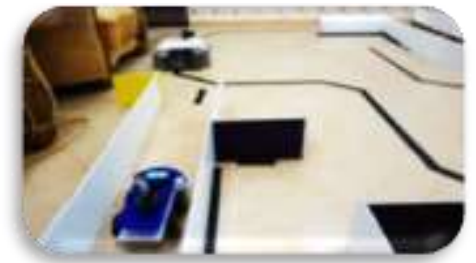

$(\mathrm{g})$

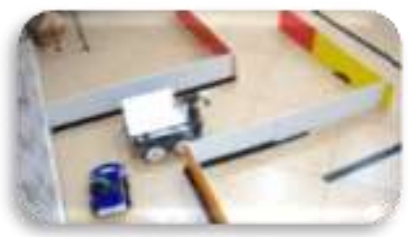

(i)

Fig. 12. a. The multifunction scenario b. Start the scenario c. Detecting an object d. Throwing the object in the specific area e \& f. Detecting $15 \mathrm{~cm}$ sign g. Only the slave robot going through the small area and the master robot waiting the slave robot at the end of the "15 cm" zone h. Detecting $50 \mathrm{~cm}$ sign i. Both robots going through the $50 \mathrm{~cm}$ zone.

The implementation of the two scenarios showed the ability of the proposed intelligent RGC. The two WMRs have the ability to pass different types of obstacles until they reach to the final destination. This give the capability of using this technique in different situations and different environments Indoor/Outdoor. For example it can be used in: Hospitals, Pharmacies, Factories, areas affected by disasters, ....etc.

\section{VI.CONCLUSION}

The paper proposed one of the solutions for the road intersection problem. The proposed intelligent RGC technique was empirically investigated, analyzed and verified through simulation and implementation. The aim of the project is to design two cooperative WMRs as a prototype for a smart cars. The two WMRs have the abilities to communicate with each other's. The four-wheel skid- steering design gave the robot more torque power. The navigation and the obstacle avoidance has been accomplished by intelligent guidance commands technique depending on various types of sensors. The sensors have been proven to work well by enabling the robot to detect any objects in any given direction and also it have the ability to measure the distance up to approximately 4 meters., the program code written for the Arduino wascapable of moving the robots through the area without hitting any walls and tracking the black line, and the master robot had the ability to remove the obstacle from the ground through manipulator arm . Both of the robots have the ability to send and receive information and orders from each other or from any third party like another robot or a computer. 


\section{ISO 3297:2007 Certified}

Vol. 5, Issue 5, May 2017

\section{REFERENCES}

[1] P. Ogren, E. Fiorelli, and N. E. Leonard, "Cooperative control of mobile sensor networks: Adaptive gradient climbing in a distributed environment," IEEE Transactions on Automatic Control, vol. 49, no. 8, pp. 1292-1302, 2004.

[2] F. Zhang and N. E. Leonard, "Cooperative control and filtering for cooperative exploration," IEEE Transactions on Automatic Control, vol. 55, no. 3, pp. 650-663, 2010

[3] A. I. Diveev, E. Yu. Shmalko, "Self-adjusting Control for Multi Robot Team by the Network Operator Method", 2015 European Control Conference (ECC), Linz, Austria, 978-3-9524269-3-7, 2015.

[4] Adrian EmanoilSerbencu, "Two Mobile Robotic Systems Synchronous Servicing an Assembly/Disassembly Production Line", 19th International Conference on System Theory, Control and Computing (ICSTCC), CheileGradistei, Romania, 978-1-4799-8481-7, IEEE, 2015.

[5] Lu Lu, Jie You, and Wencen Wu, "Constrained Fast Source Seeking Using Two Nonholonomic Mobile Robots", 2016 American Control Conference (ACC), Boston, MA, USA, 978-1-4673-8682-1, 2016.

[6] Mohammed Al Khalidy, Rami Maher, and Mohammed Zeki, "Wheeled Mobile Robot", Book, Lambert Academics Publishing GmbH \& Co. KG, Germany, 2012.

[7] Tianmiao Wang, Yao Wu *, Jianhong Liang, Chenhao Han, Jiao Chen and Qiteng Zhao, " Analysis and Experimental Kinematics of a SkidSteering Wheeled Robot Based on a Laser Scanner Sensor”, Sensors 2015, 15, 9681-9702; doi:10.3390/s150509681, ISSN 1424-8220, 2015.

[8] Xiaodong $\mathrm{Wu}$, Min Xu, and Lei Wang, "Differential Speed Steering Control for Four-Wheel Independent Driving Electric Vehicle", International Journal of Materials, Mechanics and Manufacturing, Vol. 1, No. 4, November 2013.

[9] Mohammed Al Khalidy, "Nonholonomic Wheeled Mobile Robot Modeling and Development", IEEE, 2011.

[10] Wong, J., Y., Theory of Ground Vehicles, John Wiley \& Sons, Inc., New York, NY,1993.

[11] Roland Siegwart and Illah R. Nourbakhsh, "Introduction to Autonomous Mobile Robots", A Bradford Book, The MIT Press, Cambridge, London, England, Massachusetts Institute of Technology, 2004.

[12] E. A. Lee and S. A. Seshia, "Introduction to Embedded Systems - A Cyber-Physical Systems Approach", book, First Edition, Version 1.06, LeeSeshia.org, 2011.

[13] SašoBlažič, "A novel trajectory-tracking control law for wheeled mobile robots”, Elsevier, Robotic and Autonomous Systems, 59-1001-1007, 2011

[14] Walter, W. Grey, "A Machine that Learns," Scientific American, , p60-63, August 1951.

[15] Bill Earl,"Pixy Pet Robot - Color vision follower", IEEE pp 4, june 6, 2015.

[16] Ms. Sonal J. Rane, "A Simulation Study of Behaviour of Mobile Zigbee Node”, IEEE pp. 1, September 2012

[17] Spyros G.Tzafestas, "Microprocessors in Signal Processing, Measurement and Control", Book, ISBN13: 9789027714978, Springer, Dordrecht, Netherlands, pp. 346, 1983

[18] M. Nitulescu, "Theoretical Aspects in Wheeled Mobile Robot Control", 1-4244-2577-IEEE, 2008.

[19] S. Shafer, "Using color to separate reflection components", COLOR research and application, vol. 10, pp. 210-218, Winter 1985.

[20] Ranky, P. G., C. Y. Ho, "Robot Modeling, Control \& Applications", IFS Publishers, Springer, UK, 2005.

[21] SainathGunjikar, ShivanandBorgalli, RudrappaGujanatti, and BasavarajChougala, "Multi Robot Communication for Industrial Automation", International Journal of Innovative Research in Computer and Communication Engineering, Vol. 4, Issue 6, June 2016.

\section{BIOGRAPHY}

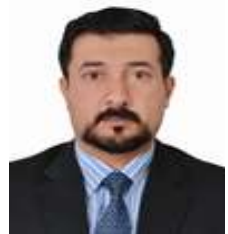

Mohammed Al Khalidy received his M.Sc., Electrical and Electronics Engineering, University of Technology, Iraq, 2002 and his Ph.D. in Electrical and Electronics Engineering, University of Technology, Iraq, 2007.He was Senior Project Engineer, Al-Karama Public Company (Industrial Company), Iraq, Baghdad, 1997- 2006. Deputy Director for Electrical Department and an Assistant Professor with the Electrical Engineering Department, Al-Mustansiryah University, College of Engineering, Iraq, Baghdad, 2006 - 2009. Assistant Professor with the Electrical and Electronics Engineering Department and a Manager of Academic Accreditation Unit, GU, 2009-2014. Since 2014, he has been an Assistant Professor with the Electrical and Electronics Engineering Department, University of Bahrain, Engineering College. He has many published researches, In 2012 he published his first book "Wheeled Mobile Robot", Lambert Academics Publishing GmbH \& Co. KG. His research interests include Electronics, Intelligent Systems and Robotics, Aerospace control and Guidance Systems. Image processing and Sustainable Energy. 